\title{
Herdabilidade de características de produção e postura em matrizes de codornas de corte
}

\author{
Heritability of production and laying traits in meat-type quails
}

\author{
Bruno Bastos Teixeira ${ }^{\mathrm{I} *}$ Ricardo Frederico Euclydes ${ }^{\mathrm{I}}$ Rafael Bastos Teixeira ${ }^{\mathrm{II}}$ \\ Luciano Pinheiro da Silva ${ }^{\mathrm{I}}$ Robledo de Almeida Torres ${ }^{\mathrm{I}}$ Felipe Gomes da Silva ${ }^{\mathrm{I}}$ \\ Helmut Gonçalves Lehner ${ }^{I}$ Giovani da Costa Caetano ${ }^{\mathrm{I}}$
}

\section{RESUMO}

\begin{abstract}
Objetivou-se no presente trabalho determinar herdabilidades de características de desempenho produtivo e reprodutivo de diferentes grupos genéticos de matrizes de codornas de corte, estimando os parâmetros genéticos por meio de análises univariadas. Foram avaliados dois grupos genéticos (UFV1 e UFV2), no período de 2006 a 2009, sendo acompanhadas cinco gerações, totalizando 2136 matrizes. As codornas foram pesadas ao nascimento, $7,14,21,28,35,42$, 77, 112 e 147 dias de idade. Foram avaliados ovos de cada codorna, coletados por três dias consecutivos, durante quatro períodos de 35 dias e medidos a largura e comprimento dos ovos, pesos de gema, casca e albúmen. Também foi avaliada a gravidade específica dos ovos e mensuradas a taxa de postura total e a idade ao primeiro ovo. Foram realizadas análises univariadas para estimação dos componentes de variância, utilizando-se o método da máxima verossimilhança restrita, por meio do programa MTDFREML e calculadas as hedabilidades. Para as características de qualidade dos ovos, as herdabilidades apresentaram valores de moderados a altos $(0,13$ à 0,55$)$, valores elevados para crescimento $(0,64$ à 0,68$)$ e moderados a alto para forma do ovo $(0,10$ à 0,57$)$. As estimativas de herdabilidades encontradas para qualidade dos ovos indicam uma alta variabilidade genética para ambos os grupos genéticos. Já para os pesos, indicaram que a seleção dentro da população poderia resultar em aumentos dos pesos por meio da seleção baseada na informação individual. Para as características de forma de ovo, os valores de herdabilidade sugerem a possibilidade de grupos genéticos que atendam a um padrão de forma.
\end{abstract}

Palavras-chave: Coturnix coturnix, grupos genéticos, parâmetros genéticos.

\section{ABSTRACT}

The objective of this study to determine the heritability of characteristics of productive and reproductive performance of different genetic groups of arrays of meat quails, estimating genetic parameters using univariate analyzes. We evaluated two genetic groups (UFV1 and UFV2) in the period 2006 to 2009, being accompanied by five generations, totaling 2136 arrays. Quail were weighed at birth, 7, 14, 21, 28, 35, 42, 77, 112 and 147 days of age. We evaluated each quail eggs were collected for three consecutive days, during four periods of 35 days and measured the width and length of eggs, yolk weight, albumen and shell. We evaluated the specific gravity of eggs and measured the rate of overall posture and age at first egg. Univariate analyzes were performed to estimate the variance components, using the method of restricted maximum likelihood using the program and calculated the MTDFREML hedabilidades. For the quality of eggs heritability values were moderate to high (0.13 to 0.55), high values for growth (0.64 to 0.68 ) and moderate to high for egg shape (0.10 to 0.57). Heritability estimates for egg quality found indicate a high genetic variability for both genetic groups. As for the weights indicated that selection within the population could result in increases in weight by selection based on individual information. For the characteristics of an egg shape, the heritability values suggest the possibility of genetic groups that meet a standard shape.

Key words: Coturnix coturnix, genetic groups, genetic parameters.

\section{INTRODUÇÃO}

Um ramo da avicultura que tem atraído interesse é a coturnicultura. Essa atividade possibilita o retorno do capital investido, além de ser uma alternativa para a alimentação humana. Seus principais produtos são a carne e os ovos, de grande aceitação no mercado.

No Brasil, o crescimento da coturnicultura tem encontrado barreiras que dificultam a exploração e

'Departamento de Zootecnia, Universidade Federal de Viçosa (UFV), 36571-000, Viçosa, MG, Brasil. E-mail: brunobatzootecnia@yahoo.com.br. *Autor para correspondência.

"Departamento de Zootecnia, Instituto Federal Minas Gerais, Campus Bambuí, MG, Brasil. 
maximização da produção. Uma dessas dificuldades é a falta de estudo dos potenciais produtivos sobre os principais grupos genéticos disponíveis. Por isso, tornase necessária a implantação de programas de melhoramento genético, que busquem acompanhar o desenvolvimento e a evolução das características de importância econômica.

Para se obter material genético de qualidade, são necessários programas de melhoramento bem estruturados, que necessitam de constante acompanhamento das características de importância econômica de cada linhagem (TEIXEIRA et al., 2012). Para isso, a avaliação da estrutura das populações disponíveis por meio da estimação dos parâmetros genéticos é fundamental para se definirem as estratégias de seleção.

Nota-se, também, que muitos dos trabalhos encontrados na literatura (STRONG et al., 1978; NESTOR et al., 1982; BAUMGARTNER, 1994), com relação à estimação de parâmetros genéticos em codornas, são baseados em metodologias utilizadas nas décadas de 60 e 70, que forneciam estimativas viesadas, tais como o método dos mínimos quadrados. Atualmente, com o avanço tecnológico, é possível obter estimativas mais acuradas, por meio de modelo animal, e se estimar parâmetros genéticos por meio de máxima verossimilhança restrita, além de, consequentemente, se obter predições mais confiáveis.

Objetivou-se no presente trabalho determinar herdabilidades de características de desempenho produtivo e reprodutivo de diferentes grupos genéticos de matrizes de codornas de corte, estimando os parâmetros genéticos por meio de análises univariadas.

\section{MATERIAL E MÉTODOS}

Foram avaliados dois grupos genéticos, de origens distintas de codorna de corte, provenientes do programa genético da UFV, denominados UFV1 e UFV2. Utilizaram-se informações de 2136 matrizes, sendo 1026 provenientes do grupo genético UFV1 e 1110 do grupo UFV2. Os dados foram coletados de 2006 a 2009, durante cinco gerações, sendo que em cada geração foram acasaladas ao acaso 204 matrizes com 102 machos.

As codornas foram pesadas ao nascimento, 7, 14, 21, 28, 35, 42, 77, 112 e 147 dias de idade. Foram avaliados ovos de cada ave, coletados por três dias consecutivos, durante quatro períodos de 35 dias, a partir do $42^{\circ}$ dia de idade, sendo que as características utilizadas na análise foram: peso médio do ovo (POM), peso médio da casca $(\mathrm{PCM})$, peso médio da gema (PGM), gravidade específica média do ovo (DM), largura média do ovo (LOM1, LOM2, LOM3 e LOM4), comprimento médio do ovo (COM1, COM2, COM3 e COM4), taxa de postura total (TXT) e idade ao primeiro ovo (IDPO), em que os números 1, 2, 3 e 4 representam os períodos de 1 a 35,36 a 70,71 a 105 e 106 a 140 dias de postura.

A gravidade específica foi obtida por meio de densímetro de óleos minerais, com escala de 1,050/ 1,100. Para avaliar a gravidade específica dos ovos, foram retirados todos os ovos íntegros produzidos a partir do $17^{\circ}$ dia de cada período e avaliados em oito soluções de $\mathrm{NaCl}$, variando de 1,055 a $1,090 \mathrm{~g} \mathrm{~cm}^{-3}$. O peso médio dos ovos, em gramas, foi obtido pela coleta e pesagem em balança de precisão de $0,01 \mathrm{~g}$, durante três dias consecutivos, a partir do $14^{\circ}$ dia do período de avaliação da produção, em quatro períodos de 35 dias.

Após a pesagem, os ovos foram cortados com estilete e suas gemas separadas e pesadas. As cascas identificadas foram secas e pesadas, obtendose, assim, os pesos das cascas. $\mathrm{O}$ formato do ovo altera a resistência física da casca e o índice utilizado para avaliar o formato do ovo é a relação largura e altura. Para mensuração da largura e altura dos ovos, foi utilizado um paquímetro digital.

Foram realizadas análises univariadas para estimação dos componentes de variância, utilizandose o método da máxima verossimilhança restrita. Essa abordagem foi escolhida devido à dificuldade de convergência em análises multicaracterísticas. As estimativas de herdabilidades foram calculadas separadamente para cada grupo genético, sem visar a sua comparação. $\mathrm{O}$ modelo estatístico utilizado na análise das características considerou o efeito fixo de geração-eclosão e os efeitos aleatórios genético aditivo direto e residual. Esses modelos podem ser representados, na forma matricial, por $y=X \beta+Z a+e$, em que: $\mathrm{y}$ representa o vetor de observações; $X$ a matriz de incidência dos efeitos fixos; $\beta$ o vetor de efeitos fixos; $\mathrm{Z}$ a matriz de incidência dos efeitos aleatórios genéticos aditivos diretos; $a$ o vetor de efeitos aleatórios genéticos aditivos diretos; e $e$ o vetor de resíduos aleatórios. As pressuposições assumidas para os efeitos aleatórios foram as seguintes:

$$
\left[\begin{array}{l}
a \\
e
\end{array}\right] \sim N M V\left\{\left[\begin{array}{l}
0 \\
0
\end{array}\right],\left[\begin{array}{ll}
G & \phi \\
\phi & R
\end{array}\right]\right\} \text {, em que: }
$$

$G=A \sigma_{a}^{2}$ é a matriz de (co)variâncias genéticas aditivas, sendo $\mathrm{A}$ a matriz de numeradores do coeficiente de parentesco e $\sigma_{a}^{2}$ a variância genética aditiva; $R=I \sigma_{e}^{2}$ a matriz de variância residual, sendo I a matriz identidade e $\sigma_{e}^{2}$ a variância residual.

As estimativas dos componentes de (co)variância e dos parâmetros genéticos foram obtidas pelo programa MTDFREML (Multiple Trait DerivativeFree Restricted Maximum Likelihood), descrito por BOLDMAN et al. (1995), que utilizaram a metodologia da máxima verossimilhança restrita livre de derivadas 
(DFREML). O MTDFREML utiliza o algoritmo simplex para localizar o mínimo de $-2 \log _{\mathrm{e}} \mathrm{L}$ ( $\mathrm{L}=$ função de verossimilhança), e os componentes de (co)variância que minimizam a função $-2 \log _{\mathrm{e}} \mathrm{L}$ são estimativas de máxima verossimilhança.

Como critério de convergência, utilizou-se a variância dos valores do simplex $\left(-2 \log _{\mathrm{e}} \mathrm{de}\right.$ verossimilhança), menores do que $10^{-9}$. Após cada convergência, o programa foi reiniciado, usando as estimativas obtidas anteriormente, como valores iniciais. Esse procedimento foi repetido até que as diferenças entre as estimativas das duas últimas convergências fossem menores que $10^{-4}$.

\section{RESULTADOS E DISCUSSÃO}

Características de qualidade e produção de ovos

As estimativas de herdabilidade das análises univariadas da média do peso do ovo (POM), da gema (PGM), casca (PCM), albúmen (PAM), densidade específica (DM), da taxa de postura (TXT) e idade ao primeiro ovo (IDPO) das matrizes de corte dos grupos genéticos UFV1 e UFV2, nos diferentes períodos (1 ${ }^{\mathrm{a}}$, $2^{\underline{a}}, 3^{\underline{a}}$ e $\left.4^{\mathrm{a}}\right)$, são apresentadas na tabela 1 .

A herdabilidade das características de qualidade dos ovos apresentaram valores de moderados a altos para ambos os grupos genéticos, sendo POM de
0,17 a 0,55, PGM 0,13 a 0,37, PCM 0,21 a 0,49, PAM 0,23 a 0,55 e DM 0,15 a 0,44. Tal resultado indica que, além do fato de essas codornas terem sua seleção focada apenas para a característica de peso corporal, apresentam ainda alto controle genético em relação a aspectos de qualidade dos ovos, sendo isso um fato favorável para se realizar seleção com base no fenótipo.

Resultados semelhantes foram obtidos por SILVA et al. (2007), que, ao avaliarem três linhagens de codornas japonesas, observaram valores de coeficientes de herdabilidade de 0,32 a 0,43 para peso do ovo, aos 70 dias, tendo, contudo, seu valor aumentado de 0,40 a 0,54, quando avaliado aos 100 dias de idade. No entanto, sabe-se que as codornas japonesas estão submetidas há décadas à seleção, buscando melhorar a qualidade e a produção de ovos, o que resultou numa diminuição da variabilidade genética para essas características, gerando ganhos genéticos cada vez menores, como, por exemplo, para peso de ovo aos 90 dias.

As estimativas de herdabilidades, de modo geral, foram superiores a 0,40 para PCM, demonstrando outro aspecto a receber atenção por parte dos produtores e pesquisadores, que seria em relação à eclodibilidade dos ovos. A grande variabilidade existente para essas características pode ser a responsável pela variação no tempo de incubação e no nascimento das

Tabela 1 - Estimativas de herdabilidade da média do peso do ovo (POM), da gema (PGM), da casca (PCM), do albúmen (PAM), da densidade específica (DM), da taxa de postura (TXT) e idade ao primeiro ovo (IDPO) de matrizes de corte dos grupos genéticos UFV1 e UFV2, nos diferentes períodos avaliados $\left(1^{\underline{a}}, 2^{\underline{a}}, 3^{\underline{a}}\right.$ e $\left.4^{\underline{a}}\right)$.

\begin{tabular}{|c|c|c|c|c|c|}
\hline Características & Grupo genético & $1^{\circ}$ Período & $2^{\circ}$ Período & $3^{\circ}$ Período & $4^{\circ}$ Período \\
\hline \multirow{2}{*}{ POM } & UFV1 & $0,27 \pm 0,08$ & $0,30 \pm 0,09$ & $0,54 \pm 0,2$ & $0,43 \pm 0,13$ \\
\hline & UFV2 & $0,17 \pm 0,03$ & $0,36 \pm 0,1$ & $0,55 \pm 0,23$ & $0,40 \pm 0,12$ \\
\hline \multirow{2}{*}{ PGM } & UFV1 & $0,13 \pm 0,06$ & $0,21 \pm 0,05$ & $0,21 \pm 0,09$ & $0,26 \pm 0,04$ \\
\hline & UFV2 & $0,18 \pm 0,05$ & $0,30 \pm 0,12$ & $0,37 \pm 0,09$ & $0,33 \pm 0,07$ \\
\hline \multirow{2}{*}{ PCM } & UFV1 & $0,41 \pm 0,18$ & $0,46 \pm 0,1$ & $0,38 \pm 0,08$ & $0,47 \pm 0,14$ \\
\hline & UFV2 & $0,21 \pm 0,13$ & $0,33 \pm 0,09$ & $0,46 \pm 0,1$ & $0,49 \pm 0,12$ \\
\hline \multirow{2}{*}{ PAM } & UFV1 & $0,38 \pm 0,08$ & $0,42 \pm 0,07$ & $0,54 \pm 0,2$ & $0,54 \pm 0,19$ \\
\hline & UFV2 & $0,25 \pm 0,9$ & $0,29 \pm 0,08$ & $0,55 \pm 0,1$ & $0,35 \pm 0,18$ \\
\hline \multirow{2}{*}{ DM } & UFV1 & $0,31 \pm 0,12$ & $0,15 \pm 0,04$ & $0,36 \pm 0,08$ & $0,31 \pm 0,15$ \\
\hline & UFV2 & $0,18 \pm 0,09$ & $0,16 \pm 0,03$ & $0,44 \pm 0,09$ & $0,22 \pm 0,09$ \\
\hline \multirow{2}{*}{ TXT } & UFV1 & \multicolumn{4}{|c|}{$0,1 \pm 0,03$} \\
\hline & UFV2 & \multicolumn{4}{|c|}{$0,03 \pm 0,001$} \\
\hline \multirow{2}{*}{ IDPO } & UFV1 & \multicolumn{4}{|c|}{$0,16 \pm 0,04$} \\
\hline & UFV2 & \multicolumn{4}{|c|}{$0,1 \pm 0,03$} \\
\hline
\end{tabular}


codorninhas, que varia de 17 a 21 dias. As herdabilidades encontrados para PCM de ambos os grupos genéticos, demonstram a possibilidade de se poder melhorar a qualidade de casca, por meio da seleção e, consequentemente, diminuir a quebra dos ovos durante o transporte, além de promover maior eclodibilidade.

Observaram-se estimativas de herdabilidade de média a alta, para a característica $\operatorname{DM}(0,15$ a 0,44$)$. Deve-se lembrar que a variabilidade em relação ao peso do ovo influencia na sua densidade. À medida que o peso dos ovos se torna mais homogêneo, diminui-se a variabilidade da densidade, consequentemente.

As herdabilidades obtidas para IDPO $(0,10$ e 0,16$)$ se devem à influência do meio ambiente nessa característica. Dentre os fatores de ambientes relacionados a essas características, estão: época de nascimento, nutrição, programa de luz, peso corporal, etc. Outro fator que influencia a IDPO é o fato de essas matrizes serem alojadas em gaiolas aos 28 dias de idade, que torna o manejo mais homogêneo e reduz a variabilidade para essa característica.

Também foram encontradas baixas estimativas de herdabilidade para TXT $(0,03$ e 0,1$)$, o que indica que a produção apresenta baixo potencial de ganhos genéticos por meio de seleção. SILVA et. al. (2007), ao estudarem codornas de postura, encontraram valores de herdabilidade para produção de ovos até aos 90 dias praticamente nulos.

HIDALGO et al. (2007), ao estudarem codornas de postura, citaram que as estimativas de herdabilidade para peso dos ovos $(0,24$ a 0,47$)$ foram menores que as de pesos corporais $(0,78$ a 0,85$)$ sendo que a primeira decresceu à medida que a idade avançou. Esses resultados mostram que a variabilidade genética para peso do ovo tende a se reduzir ao longo do período de produção. Tal aspecto não foi observado nesse estudo com codornas europeias, pois as matrizes mantiveram um crescimento corporal até os 147 dias de idade, o que justifica os valores de herdabilidade encontrados.

Características de crescimento

As estimativas de herdabilidade do peso corporal das codornas ao nascimento, P7, P21, P28,
P35, P42, P77, P112 e P147 dias de idade das análises univariadas das matrizes de codorna de corte dos grupos genéticos UFV1 e UFV2, são apresentadas na tabela 2. Observaram-se elevados valores de herdabilidade para peso ao nascimento $(0,64$ e 0,68$)$, o que indica um potencial de resposta à seleção massal.

Dos 7 aos 42 dias, as estimativas de herdabilidade do peso corporal variaram de 0,26 a 0,44 para os dois grupos genéticos, sendo consideradas de média a alta. WINTER (2005) verificou, em codornas de corte, herdabilidades semelhantes para peso aos 7 , 14,28 e 42 dias, sendo 0,$25 ; 0,43 ; 0,53$ e 0,62 , respectivamente. Estimativas de herdabilidades de média a alta para pesos corporais, indicam uma razoável resposta à seleção individual.

AGGREY \& CHENG (1994), ao avaliaremem codornas japonesas o peso corporal no nascimento, aos 7, 14, 21 e 28 dias de idade, utilizando o método de máxima verossimilhança restrita, livre de derivadas, encontraram estimativas de herdabilidade de 0,$38 ; 0,12$; 0,$31 ; 0,12$ e 0,44 , respectivamente. Os autores sugerem que a seleção para os pesos ao nascimento, aos 14 e 28 dias de idade, pode ter resultados satisfatórios.

PAIVA et al. (2005) obtiveram estimativas de herdabilidade para os pesos ao nascimento, 7, 14, 21, 28 e 35 dias de idade, respectivamente de 0,$25 ; 0,03 ; 0,13$; 0,$15 ; 0,18$ e 0,24 , indicando que a seleção deve ser feita com base na predição do valor genético e por meio do modelo animal, usando toda informação disponível.

Observa-se também que, apesar do ambiente homogêneo proporcionado nas gaiolas, ainda há variabilidade nos pesos obtidos nos períodos. Isso mostra que, ao longo do ciclo de postura das codornas, elas ainda não estabilizaram o seu crescimento, com isso, há variabilidade genética para ganho de peso ao longo do ciclo de postura.

Características de forma do ovo

As estimativas de herdabilidade da largura média do ovo (LOM) e do comprimento médio do ovo (COM) das análises univariadas das matrizes de codorna de corte dos grupos genéticos UFV1 e UFV2 são apresentadas na tabela 3 . Observaram-se valores

Tabela 2 - Estimativas de herdabilidade $\left(h^{2}\right)$ do peso corporal das codornas ao nascimento, P7, P21, P28, P35, P42, P77, P112 e P147 dias de idade das análises univariadas das matrizes de codorna de corte dos grupos genéticos UFV1 e UFV2.

\begin{tabular}{lllllllllll}
\hline$h^{2}$ & $\mathrm{P}_{0}$ & $\mathrm{P} 7$ & $\mathrm{P} 14$ & $\mathrm{P} 21$ & $\mathrm{P} 28$ & $\mathrm{P} 35$ & $\mathrm{P} 42$ & $\mathrm{P} 77$ & $\mathrm{P} 112$ & P147 \\
\hline \multirow{2}{*}{ UFV1 } & 0,64 & 0,30 & 0,35 & 0,42 & 0,40 & 0,35 & 0,32 & 0,32 & 0,22 & 0,32 \\
& $\pm 0,2$ & $\pm 0,1$ & $\pm 0,1$ & $\pm 0,01$ & $\pm 0,2$ & $\pm 0,12$ & $\pm 0,1$ & $\pm 0,1$ & $\pm 0,08$ & $\pm 0,1$ \\
& & & & & & & & & & \\
\multirow{2}{*}{ UFV2 } & 0,68 & 0,30 & 0,28 & 0,26 & 0,43 & 0,44 & 0,24 & 0,26 & 0,26 & 0,42 \\
& $\pm 0,2$ & $\pm 0,1$ & $\pm 0,1$ & $\pm 0,07$ & $\pm 0,12$ & $\pm 0,13$ & $\pm 0,08$ & $\pm 0,06$ & $\pm 0,07$ & $\pm 0,17$ \\
\hline
\end{tabular}

Ciência Rural, v.43, n.2, fev, 2013. 
Tabela 3 - Estimativas de herdabilidade $\left(h^{2}\right)$ da largura média do ovo (LOM) e do comprimento médio do ovo (COM) das análises univariadas das matrizes de codorna de corte dos grupos genéticos UFV1 e UFV2, nos diferentes períodos avaliados.

\begin{tabular}{lcccccccc}
\hline$h^{2}$ & LOM1 & LOM2 & LOM3 & LOM4 & COM1 & COM2 & COM3 & COM4 \\
\hline UFV1 & $0,17 \pm 0,05$ & $0,24 \pm 0,04$ & $0,47 \pm 0,12$ & $0,27 \pm 0,09$ & $0,10 \pm 0,06$ & $0,38 \pm 0,04$ & $0,38 \pm 0,12$ & $0,57 \pm 0,18$ \\
UFV2 & $0,25 \pm 0,08$ & $0,25 \pm 0,06$ & $0,50 \pm 0,15$ & $0,49 \pm 0,15$ & $0,15 \pm 0,06$ & $0,29 \pm 0,08$ & $0,34 \pm 0,1$ & $0,32 \pm 0,09$ \\
\hline
\end{tabular}

de herdabilidade de moderado a alto para $\operatorname{LOM}(0,17 \mathrm{a}$ $0,50)$ e $\operatorname{COM}(0,10$ a 0,57$)$, sugerindo a possibilidade de grupos genéticos que atendam a um padrão de índice de forma, o qual é calculado em função da largura e do comprimento do ovo.

As características físicas do ovo podem interagir ou influenciar nas condições requeridas na incubação. O tamanho e a forma do ovo, aliados à porosidade da casca, afetam a perda de água durante a incubação e influenciam nos requerimentos de temperatura e umidade, principalmente durante a última semana de incubação, segundo SCHMIDT et al. (2002).

\section{CONCLUSÃO}

As estimativas de herdabilidades encontradas para os aspectos de qualidade dos ovos indicam uma alta variabilidade genética para ambos os grupos genéticos. Já para os pesos, indicaram que a seleção dentro da população poderia resultar em aumentos dos pesos por meio da seleção baseada na informação individual. Para o peso ao nascimento, os valores de herdabilidade indicam um alto potencial de resposta à seleção massal. Para as características de forma de ovo, os valores de herdabilidade sugerem a possibilidade de grupos genéticos que atendam a um padrão de forma.

\section{REFERÊNCIAS}

AGGREY, S.E.; CHENG, K.M. Animal model analysis of genetic (co)variances for growth traits in Japanese quail. Poultry Science, v.73, n.12, p.1822-1828, 1994. Disponível em: <http://www.ncbi.nlm.nih.gov/pubmed/7877938>. Acesso em: 10 nov. 2008.

BAUMGARTNER, J. Japanese quail production, breeding and genetics. Poultry Science Journal, v.50, n.2, p.227-235, 1994.
BOLDMAN, K.G.A et al. A manual for use of MTDFREML. A set of programs to obtain estimates of variance and covariances. Washington, DC.: ARS, USDA, 1995. 120p.

HIDALGO, A.M. et al. Parâmetros genéticos para peso do ovo e peso corporal em codornas de zxc postura. In: SIMPÓSIO INTERNACIONAL DE COTURNICULTURA, 3.; CONGRESSO BRASILEIRO DE COTURNICULTURA, 2., 2007, Lavras, MG. Anais... Lavras: UFLA, 2007. p.219.

NESTOR, K.E. et al. Divergent selection for body weight and yolk precursor in Coturnix coturnix Japonica. Poultry Science Journal, v.61, n.3, p.12-17, 1982.

PAIVA, E. et al. Parâmetros genéticos e fenotípicos para peso aos 28 dias, idade ao primeiro ovo e peso do ovo em três linhagens de codornas de postura. In: REUNIÃO ANUAL DA SOCIEDADE BRASILEIRA DE ZOOTECNIA, 42., 2005, Goiânia, GO. Anais... Goiania: Sociedade Brasileira de Zootecnia, 2005. CD-ROM, Melhoramento Genético Animal.

SCHMIDT, G.S. et al. Fatores que afetam a qualidade do pinto de corte. Artigos Embrapa suínos e aves, 2002. Disponível em: <http://www.cnpsa.embrapa.br/?/artigos/2002/artigo-2002n018.html;ano=2002>. Acesso em: 11 set. 2008.

SILVA, R.M. et. al. Parâmetros genéticos para peso do ovo e produção de ovos em três linhagens de codorna de postura. In: SIMPÓSIO INTERNACIONAL DE COTURNICULTURA, 3.; CONGRESSO BRASILEIRO DE COTURNICULTURA, 2., 2007, Lavras, MG. Anais... Lavras: UFLA, 2007. p.213.

STRONG, C.F. et al. Inheritance of egg production, egg weight, body weight and certain plasma constituents in Coturnix. Poultry Science Journal, v.57, n.2, p.1-9, 1978.

TEIXEIRA, B.B. et al. Estimação dos componentes de variância para as características de produção e de qualidade de ovos em matrizes de codorna de corte. Ciência Rural, v.42, n.4, p.713$717,2012$.

WINTER, E.M.W. Estimação de parâmetros genéticos de características de desempenho, carcaça e composição corporal de codornas para corte (Coturnix sp.). 2005. 91f. Dissertação (Mestrado em Zootecnia) - Curso de Pós-graduação em Zootecnia Universidade Federal do Paraná, PR. 